\title{
Making weight: acute muscle weakness and hypokalaemia exacerbated by thyrotoxicosis factitia in a bodybuilder
}

\author{
Clare E Bonnar1, John F Brazil1, Julie O Okiro², Louise Giblin², Yvonne Smyth³, \\ Paula M O'Shea 4 and Francis M Finucane (D) \\ ${ }^{1}$ Centre for Diabetes, Endocrinology \& Metabolism, 2Department of Nephrology, ${ }^{3}$ Department of Cardiology, and \\ ${ }^{4}$ Department of Clinical Biochemistry, Galway University Hospitals, Saolta University Healthcare Group, \\ Galway, Ireland
}

\author{
Correspondence \\ should be addressed \\ to F M Finucane \\ Email \\ francis.finucane@nuigalway.ie \\ or francis@gmail.com
}

\section{Summary}

A 32-year-old Caucasian male presented to the emergency department with a one-day history of acute severe bilateral lower limb weakness, three days after competing in a bodybuilding competition. He consumed large quantities of carbohydrate-rich foods following the competition. His past medical history was significant for anxiety, and family history was non-contributory. Examination was normal except for reduced power and hyporeflexia in both legs, despite his muscular physique. He was noted to have severe hypokalaemia ( $\left.\mathrm{K}^{+}=1.9 \mathrm{mmol} / \mathrm{L}\right)$. His thyroid function tests were consistent with thyrotoxicosis. He reported taking thyroxine and several other agents to facilitate muscle mass generation before the bodybuilding competition. His presentation was reminiscent of thyrotoxic periodic paralysis, albeit uncommon with Caucasian ethnicity. He also had transient hyperglycaemia at presentation with concomitant hyperinsulinaemia, which could be attributed to the carbohydrate load and may have exacerbated his hypokalaemia through a transcellular shift. Urine toxicology screen subsequently ruled out the use of diuretics but confirmed the presence of a long-acting beta agonist (clenbuterol) which, along with other substances, may have aggravated the hypokalaemia further. After $12 \mathrm{~h}$ of i.v. replacement, the potassium level normalised and leg weakness resolved. The patient agreed to stop taking thyroxine and beta agonists and was well during the clinic visit at one month follow-up. This case highlights the potential for thyrotoxicosis factitia to exacerbate hypokalaemia and muscle weakness from other causes in bodybuilders presenting with acute severe weakness, irrespective of ethnicity.

\section{Learning points:}

- In patients presenting with muscle weakness and hypokalaemia, early consideration of thyrotoxicosis is essential, even in the absence of a past history of thyroid disease or specific symptoms of thyrotoxicosis, in order to allow prompt initiation of appropriate treatment and to prevent recurrence.

- Bodybuilders may constitute a uniquely 'at-risk' group for thyrotoxic periodic paralysis secondary to thyrotoxicosis factitia, especially where there is concomitant use of beta-adrenergic agonists, even in the absence of diuretic use.

- Although rare and usually described in patients of Asian or Polynesian ethnicity, this case highlights that thyrotoxic periodic paralysis secondary to thyrotoxicosis factitia can also occur in patients with Caucasian ethnicity.

- We speculate that consuming large quantities of carbohydrates may induce hyperinsulinaemia, which could theoretically contribute to worse hypokalaemia, though mechanistic studies would be needed to explore this further. 


\section{Background}

Thyrotoxic periodic paralysis (TPP) is a rare endocrine emergency, first described in Japan in 1957 (1), which presents with a triad of thyrotoxicosis, hypokalaemia and muscle weakness (2). It is usually associated with underlying thyroid disease, most commonly Graves' thyrotoxicosis (3). There is a male preponderance and patients invariably have a non-White ethnic background: In one singlecentre study in the US describing 19 patients presenting with 24 episodes of TPP over 15 years, all were men, one was of Black African ethnicity and the rest were of Asian (Korean, Filipino, Hispanic and Vietnamese) ethnicity (4). Hypokalaemia and paralysis are thought to arise from overstimulation of Na-K-ATPase, with potassium influx into the skeletal muscle leading to disruption of the functioning of the skeletal muscle-specific inward rectifying potassium channel and paradoxical depolarisation which in turn inactivates sodium channels, leading to paralysis (5). Although this overstimulation of Na-K-ATPase is driven by excess thyroid hormone, the clinical manifestations of thyrotoxicosis in these patients are often mild (3). Acute episodes of paralysis in TPP can by precipitated by the ingestion of large quantities of carbohydrates (4), probably due to further stimulation of potassium sequestration in skeletal muscle by insulin (5). Treatment of acute presentations of this rare and potentially fatal condition requires emergency measures to replace the depleted potassium and a high degree of clinical suspicion for underlying thyroid disease in patients presenting with weakness and hypokalaemia.

\section{Case presentation}

A 32-year-old male bodybuilder presented to our emergency department overnight, with a 24-h history of progressively worsening weakness in both legs and difficulty in walking. Two days previously, he had participated in an international bodybuilding competition, immediately after which he had consumed three very large carbohydrate-rich meals. There was no family or past medical history of note, except moderately severe anxiety of four years duration, which his general practitioner had attributed to thyroxine use. Thyrotoxicosis had been confirmed by his general practitioner with fully suppressed thyroid stimulating hormone (TSH) on two sets of thyroid function tests previously, but the patient continued to take thyroxine against medical advice, which he obtained through the internet. In addition, he reported taking growth hormone, anabolic steroids (including trenbolone, stanozolol, oxandrolone, mesterolone, fluoxymesterone and drostanolone), a long-acting $\beta 2$-agonist (clenbuterol) and a non-steroidal aromatase inhibitor (letrozole), all sourced illicitly online. He was married with two children, did not drink or smoke and went to the gym every day to lift weights. On systems review, aside from anxiety and muscle weakness, he had no other symptoms of thyrotoxicosis. He had no sensory symptoms or urinary or faecal incontinence. He denied liquorice ingestion, tobacco-chewing, diuretic or laxative abuse.

On examination he was alert and oriented, with normal affect. He was apyrexial and haemodynamically stable. He had a notably muscular physique. Respiratory, cardiovascular and abdominal examinations were normal and there was no goitre or features of hyperthyroidism. Neurological examination of his cranial nerves and upper limbs was normal, but he had symmetrically reduced grade $3 / 5$ power in the lower limbs (for each of hip, knee and ankle flexion and extension and hip adduction and abduction), with normal tone. He had diminished deeptendon reflexes at the knees and ankles and bilateral flexor plantar responses.

\section{Investigation}

Initial biochemical investigations revealed metabolic alkalosis and severe hypokalaemia, with normal magnesium, calcium and renal function (see Table 1). His 12-lead ECG showed low-voltage P- and T-waves, prominent $\mathrm{U}$-waves and a prolonged QT interval of $520 \mathrm{~ms}$. Creatine kinase was elevated, consistent with his increased

Table 1 Initial biochemistry results at presentation.

\begin{tabular}{|c|c|c|}
\hline Laboratory test (units) & Result & $\begin{array}{c}\text { Reference } \\
\text { range }\end{array}$ \\
\hline * pH & 7.53 & $7.35-7.45$ \\
\hline${ }^{*} \mathrm{pCO}_{2}, \mathrm{kPa}$ & 4.2 & $4.6-6.0$ \\
\hline${ }^{*} \mathrm{HCO}_{3}^{-}, \mathrm{mmol} / \mathrm{L}$ & 26.2 & $19-24$ \\
\hline *Base excess, mmol/L & 4.0 & -2.0 to +3.0 \\
\hline Sodium, mmol/L & 141 & $136-145$ \\
\hline Potassium, mmol/L & 1.9 & $3.5-4.5$ \\
\hline Phosphate, mmol/L & 0.41 & $0.87-1.45$ \\
\hline Corrected calcium, mmol/L & 2.24 & $2.17-2.51$ \\
\hline Magnesium, mmol/L & 0.68 & $0.65-1.05$ \\
\hline Urea, mmol/L & 4.6 & $2.1-7.1$ \\
\hline Creatinine, $\mu \mathrm{mol} / \mathrm{L}$ & 73 & $64-104$ \\
\hline Creatine kinase, U/L & 3128 & $39-308$ \\
\hline Plasma beta-hydroxybutyrate, mmol/L & 0.1 & \\
\hline Alanine aminotransferase, U/L & 249 & $0-40$ \\
\hline Alkaline phosphatase, U/L & 144 & $40-129$ \\
\hline Gamma glutamyltransferase, U/L & 149 & $10-71$ \\
\hline
\end{tabular}

*Arterial blood gas results $3 \mathrm{~h}$ after initial presentation. 
muscle mass and recent participation in the bodybuilding competition. His troponin $\mathrm{T}$ was elevated at $32 \mathrm{ng} / \mathrm{L}$ (normal <14) and remained so throughout the admission, suggesting myocardial injury. His random plasma glucose at presentation was elevated at $18.2 \mathrm{mmol} / \mathrm{L}$ and he had ' $3+$ ' glycosuria, but he had no osmotic symptoms and urine ketones were undetectable. A fasting plasma glucose of 7.4 $\mathrm{mmol} / \mathrm{L}$ with a concomitant fasting plasma insulin of 276 $\mathrm{pmol} / \mathrm{L}$ were consistent with hyperglycaemia secondary to insulin resistance. Thereafter, his glucose levels normalised quickly without specific treatment and his $\mathrm{HbA1c}$ was normal at $34 \mathrm{mmol} / \mathrm{mol}$. He had a mild transaminitis consistent with non-alcoholic fatty liver disease. A urinary potassium was sent in order to calculate a transtubular potassium gradient, which was low (Table 2) excluding renal potassium loss in general and diuretic use in particular as a likely cause of hypokalaemia. His thyroid function tests confirmed thyrotoxicosis, with fully suppressed TSH, although his free thyroxine and tri-iodothyronine were not elevated. Serum cortisol, testosterone, androstenedione, dihydroepiandosterone, growth hormone and serum IGF-1 were all within normal reference ranges. However, luteinising- and follicle stimulating hormone levels were undetectable and oestradiol was elevated, consistent with androgen excess secondary to his illicit anabolic steroid use. A qualitative urine drug screen, using ultraperformance liquid chromatography 'time of flight' mass spectrometry (UPLC TOF MS) confirmed the absence of diuretics or laxatives (specifically Rhein, Danthron,

Table 2 Endocrine and metabolic work-up after presentation.

\begin{tabular}{|c|c|c|}
\hline Laboratory test & Result & $\begin{array}{c}\text { Reference } \\
\text { range }\end{array}$ \\
\hline Thyroid stimulating hormone, mIU/L & $<0.02$ & $0.27-4.20$ \\
\hline Free thyroxine, pmol/L & 12.0 & $10.5-22.0$ \\
\hline Tri-iodothyronine, $\mathrm{nmol} / \mathrm{L}$ & 1.02 & $1.28-2.33$ \\
\hline Follicle stimulating hormone, IU/L & $<0.5$ & $1.5-12.4$ \\
\hline Luteinising hormone, IU/L & $<0.5$ & $1.7-8.6$ \\
\hline Oestradiol, pmol/L & 224 & 99-192 \\
\hline Testosterone, $\mathrm{nmol} / \mathrm{L}$ & 23.7 & $9.9-27.8$ \\
\hline Androstenedione, $\mathrm{nmol} / \mathrm{L}$ & 2.7 & $2.0-5.4$ \\
\hline Dihydroepiandosterone, $\mu \mathrm{mol} / \mathrm{L}$ & 3.6 & $2.3-10.0$ \\
\hline Sexhormone-binding globulin, $\mathrm{nmol} / \mathrm{L}$ & 8.4 & $14.5-48.4$ \\
\hline Cortisol, $\mathrm{nmol} / \mathrm{L}$ & 513 & $166-507$ \\
\hline IGF-1, $\mu \mathrm{g} / \mathrm{L}$ & 79 & $71-241$ \\
\hline Urine potassium, $\mathrm{mmol} / \mathrm{L}$ & 17 & \\
\hline Urine osmolality, $\mathrm{mmol} / \mathrm{kg}$ & 587 & \\
\hline Serum potassium, $\mathrm{mmol} / \mathrm{L}$ & 2.6 & $3.5-4.5$ \\
\hline Serum osmolality, mmol/kg & 284 & $275-299$ \\
\hline Trans-tubular potassium gradient & 2.84 & $<3 *$ \\
\hline
\end{tabular}

Bisacodyl and Phenolphthalein) and the presence of two of the six reported anabolic steroids (trenbolone and oxandrolone) and clenbuterol. There was no evidence to suggest either anorexia nervosa, malnutrition or chronic alcoholism (where total body content of potassium is low due to reduced intake) as causes of hypokalaemia, or any skin conditions that would lead to excessive potassium loss. He had nothing in his history to suggest primary hyperaldosteronism - there was no hypertension or hypokalaemia at previous general practioner visits in the preceding four years. He was normotensive throughout his hospital stay. There was nothing to suggest secondary hyperaldosteronism - he was not dehydrated, and had normal renal function serum osmolality. There was no evidence of ingestion of fludrocortisone or amphotericin-B, liquorice or chewing tobacco (both of which contain carbenoxolone, an 11-betaHSD1 inhibitor), nor did he have features of Cushing's syndrome. There was no family history of Barter's or Gitelman's syndromes, both of which usually present in childhood. The absence of hypokalaemia on blood tests in the preceding four years strongly refutes these diagnoses.

\section{Treatment}

The patient received i.v. fluids and potassium replacement, with serum $\mathrm{pH}$ and potassium levels returning to normal within $12 \mathrm{~h}$. Because hypokalaemia was due to an intracellular shift of potassium rather than tissue potassium loss, we were mindful of the potential for rebound hyperkalaemia and with a cautious approach and frequent monitoring, the patient required a total of only $60 \mathrm{mmol}$ potassium chloride to restore normokalaemia. The patient also received magnesium and phosphate replacement as well as prophylactic low-molecular weight heparin. He was advised to stop taking thyroxine, as well as the other illicit bodybuilding substances.

\section{Outcome and follow-up}

The patient's weakness resolved within hours of correction of his hypokalaemia. His neurological examination returned to normal. On the third day of his admission, his potassium remained normal and he was discharged home. One month later, he was followed-up in outpatients and reported stopping thyroid supplements. The echocardiogram results were normal except for mild septal hypokinesis, and a gadolinium-enhanced cardiac MRI scan showed reduced long axis motion consistent with early interstitial myocardial fibrosis from steroid use. 
His potassium and thyroid function tests were normal at that time. At two months post discharge, potassium and thyroid function tests remained normal, as were fasting glucose and insulin and HbA1c. His transaminitis had resolved and sex hormone-binding globulin (SHBG) had normalised, consistent with resolution of his liver steatosis. However, his total testosterone and oestradiol were elevated, consistent with ongoing anabolic steroid use, despite the medical advice to the contrary. He had no further episodes of limb weakness.

\section{Discussion}

This man's presentation with acute severe leg weakness and hypokalaemia was reminiscent of TPP, albeit with Caucasian ethnicity and no apparent history of thyroid disease, until systems review at presentation noted the consumption of thyroid supplements and thyrotoxicosis factitia which had been diagnosed by his general practioner. A description of a similar presentation in a 27 -year-old bodybuilder of Hispanic ethnicity published last year was only the eighth ever described of TPP secondary to thyrotoxicosis factitia (6) and we believe that ours is the first description in a patient of Caucasian ethnicity. Case series suggest that TPP is typically present in the third decade, over $95 \%$ of the time in males $(2,6)$, and is much more common in those of Asian ethnicity $(7,8)$. Our patient's presentation was typical, with rapidly progressive symmetrical muscle weakness in both legs, without sensory symptoms or signs, or bowel or bladder dysfunction $(6,7)$. Rarely, respiratory muscle involvement or ventricular arrhythmias can also occur (7). Rapid recognition and treatment of TPP is crucial and affected patients may require high dependency or intensive care unit admission. Non-selective beta blockers such as propranolol may be helpful, but we did not use this given the patient's relatively prompt improvement. In contrast to the more common condition of familial hypokalaemic periodic paralysis (FHPP, an autosomal dominant condition), the vast majority of TPP cases are sporadic, though a familial form also exists. Acute episodes of either sporadic or familial TPP are indistinguishable from those of FHPP, except that there is a higher propensity for arrhythmias, cardiac arrest and respiratory failure with the former (4). As in our case, clinical features of thyrotoxicosis may be subtle or absent in TPP (3). Unexpectedly, our patient did not have raised free thyroxine or triiodothyronine levels, which we would have anticipated given he had taken these agents $48 \mathrm{~h}$ prior to presentation. However, the presence of a fully suppressed TSH with a clear history of chronic exogenous thyroid hormone use was confirmatory of thyrotoxicosis.

Acute hypokalaemia is the hallmark of TPP and its severity correlates with the degree of muscle weakness (5), which arises from myopathy rather than neurological dysfunction (8). Other causes of hypokalaemia such as laxative or diuretic use need to be excluded, even where thyrotoxicosis is present, before TPP can be diagnosed. In particular, diuretic use may be present in bodybuilders with TPP secondary to thyrotoxicosis factitia (6), but this would be expected to lead to much greater requirements for potassium replacement, as well as an elevated trans-tubular potassium gradient and a positive urinary diuretic screen, none of which were present in our patient. Our patient's use of clenbuterol, a potent stimulator of the beta- 2 adrenergic receptor is likely to have contributed to enhanced skeletal muscle Na-KATPase activation and further sequestration of potassium (9), precipitating his acute presentation. Clenbuterol may also have contributed to hypophosphataemia and thus worsened his rhabdomyolysis. Furthermore, he may have developed a refeeding syndrome after severe dietary restriction before the bodybuilding competition, followed by high carbohydrate intake, which would have led to insulinmediated phosphate (as well as potassium) sequestration. Also, stanozolol use is the likely cause of his metabolic alkalosis and may have further aggravated his hypokalaemia (10). Acute attacks of TPP often occur when the patient is asleep or after carbohydrate-rich meals $(4,7)$, suggesting insulin may be an important contributor to intracellular potassium sequestration through its activation of Na-KATPase (5). We postulate that the significant carbohydrate binge and likely subsequent hyperinsulinemia aggravated our patient's acute presentation with hypokalaemia and weakness. His suppressed SHBG at presentation was also suggestive of insulin resistance, particularly in the context of thyroxine use, which would be expected to raise SHBG. The higher incidence of TPP in men may be due to testosterone-mediated myoblast hypertrophy, with greater expression of Na-K-ATPase (4). This patient's hypokalaemia and muscle weakness arose from a synergistic combination of exogenous thyroxine, stanazolol, clenbuterol, refeeding syndrome and anabolic steroids. The combined use of these agents by bodybuilders to gain muscle and lose fat represents a serious but underappreciated risk to health that outweighs any 'benefits' for body composition and bodybuilding competition.

\section{Declaration of interest}

Clare Bonnar, John Brazil, Julie Okiro, Yvonne Smyth, Louise Giblin and Paula O'Shea have no conflicts of interest to declare. Francis Finucane 
has (until 2017) received honoraria and travel grants and has served on advisory boards for Novo Nordisk, Eli Lilly, Ethicon, Pfizer Inc., SanofiAventis, Astra Zeneca, Merck-Serono, Boehringer Ingelheim, Janssen, and Novartis in the past.

\section{Funding}

Francis Finucane is funded by a Clinical Research Career Development Award from the Saolta University Health Care Group.

\section{Patient consent}

Fully informed, written consent was obtained from the patient to describe their care and relevant investigations in this case report for consideration for publication in EDM.

\section{Author contribution statement}

Clare Bonnar, John Brazil, Julie Okiro, Yvonne Smyth and Louise Giblin allevel contributed to the clinical care of this patient and influenced management decisions, as well as drafting and revising the manuscript. Paula O'Shea supervised the laboratory analyses and their interpretation and revised the manuscript. Francis Finucane supervised the clinical care of the patient and the drafting and revision of the manuscript.

\section{References}

1 Shizume K, Shishiba Y, Kuma K, Noguchi S, Tajiri J, Ito K \& Noh JY. Comparison of the incidence of association of periodic paralysis and hyperthyroidism in Japan in 1957 and 1991. Endocrinologia Japonica 199239 315-318. (https://doi.org/10.1507/endocrj1954.39.315)
2 Chang CC, Cheng CJ, Sung CC, Chiueh TS, Lee CH, Chau T \& Lin SH. A 10-year analysis of thyrotoxic periodic paralysis in 135 patients: focus on symptomatology and precipitants. European Journal of Endocrinology 2013169 529-536. (https://doi.org/10.1530/EJE-130381)

3 Kung AW. Clinical Review: Thyrotoxic periodic paralysis: a diagnostic challenge. Journal of Clinical Endocrinology and Metabolism 200691 2490-2495. (https://doi.org/10.1210/jc.2006-0356)

4 Manoukian MA, Foote JA \& Crapo LM. Clinical and metabolic features of thyrotoxic periodic paralysis in 24 episodes. Archives of Internal Medicine 1999159 601-606. (https://doi.org/10.1001/ archinte.159.6.601)

5 Lin SH \& Huang CL. Mechanism of thyrotoxic periodic paralysis. Journal of the American Society of Nephrology 201223 985-988. (https:// doi.org/10.1681/ASN.2012010046)

6 Patel AJ, Tejera S, Klek SP \& Rothberger GD. Thyrotoxic periodic paralysis in a competitive bodybuilder with thyrotoxicosis factitia. AACE Clinical Case Reports 20206 e252-e256. (https://doi.org/10.4158/ ACCR-2020-0154)

7 Clarine LHS \& Hosein N. Thyrotoxic periodic paralysis: a review of cases in the last decade. AACE Clinical Case Reports 20151 e182-e186. (https://doi.org/10.4158/EP14304.CR)

8 Panikkath R \& Nugent K. I lost weight, but I became weak and cannot walk - a case of nutraceutical (T3)-induced thyrotoxic periodic paralysis. American Journal of Therapeutics 201421 e211-e214. (https:// doi.org/10.1097/MJT.0b013e318288a460)

9 MacLennan PA \& Edwards RH. Effects of clenbuterol and propranolol on muscle mass. Evidence that clenbuterol stimulates muscle betaadrenoceptors to induce hypertrophy. Biochemical Journal 1989264 573-579. (https://doi.org/10.1042/bj2640573)

10 Maini AA, Maxwell-Scott H \& Marks DJ. Severe alkalosis and hypokalemia with stanozolol misuse. American Journal of Emergency Medicine 201432 196.e3-196.e4. (https://doi.org/10.1016/j. ajem.2013.09.027)

Received in final form 6 September 202

Accepted 17 September 2021 